\title{
P03-9-1 Poster session
}

\section{Cationic Functionalization of Chlorin Derivatives for Antimicrobial Photodynamic Inactivation and Related Vancomycin Conjugate}

\author{
Ahmed Elnewishy ${ }^{1,2,3}$, Liyi Huang ${ }^{2,3}$, Yingying Huang ${ }^{2,3}$, Long Chiang ${ }^{4}$, Michael Hamblin²,3,5 \\ ${ }^{1}$ Laser Applications in photochemistry, photobiology and biotechnology, The National Institute of Laser Enhanced \\ Science, Cairo University, Egypt, ${ }^{2}$ Wellman Center for Photomedicine, Massachusetts General Hospital, USA, \\ ${ }^{3}$ Department of Dermatology, Harvard Medical School, USA, ${ }^{4}$ Department of Chemistry, University of Massachusetts \\ Lowell, USA, ${ }^{5}$ Harvard-MIT Division of Health Sciences and Technology, USA
}

It is known that multiple cationic charges are required to produce broad-spectrum antimicrobial photosensitizers (PS) for photodynamic inactivation (aPDI) of bacteria and fungi. In the present study we describe the synthesis and aPDI testing of a set of derivatives prepared from the parent pheophytin molecule with different numbers of attached sidearms $(1 \& \# 8210 ; 3)$ each

consisting of five quaternized cationic groups (pentacationic). producing corresponding [Zn+2]pheophorbide-a-N (C2NC1C3)5 (Zn-Phe-N5, 5 charges), [Zn+2]chlorin e6-[N(C2NC1C3)5]2(Zn-Chl-N10, 10 charges) and [Zn+2] mesochlorin e6-[N(C2N C1C3)5]3 (Zn-mChl-N15, 15charges). Moreover, a conjugate between Zn-Phe-N5 and the antibiotic vancomycin called Van-[Zn+2]-m-pheophorbide-N(C2NC1C3)5 (Van-Zn-mPhe-N5) was also prepared. We tested these compounds against Gram-positive methicillin Staphylococcus aureus (MRSA), Gram-negative Escherichia coli, and fungal yeast Candida albicans. All three compounds were highly active against MRSA giving eradication (\&\#8805; 6 logs of killing) with $<1.0 \& \# 181$;M and $10 \mathrm{~J} / \mathrm{cm} 2$ of $415 \mathrm{~nm}$ light. The order of activity was Zn-Phe-N5 $>$ ZnChl-N10 $>$ Zn-mChl-N15. In the case of E coli the activity was much lower (eradication was only possible with 50 \&\#181; $\mathrm{M} Z \mathrm{Zn}-\mathrm{mChl}-\mathrm{N} 15$ and $20 \mathrm{~J} / \mathrm{cm} 2)$. The order of activity was the reverse of that found with MRSA (Zn-mChl-N15 $>$ ZnChlN10 > Zn-Phe-N5). Activity against C. albicans was similar to E. coli with Zn-mChl-N15 giving eradication. The activity of Van-Zn-mPhe-N5 was generally lower than Zn-Phe-N5 (except for E. coli). Red (660 nm) light was also effective as might be expected from the absorption spectra. An initial finding that Van-Zn-mPhe-N5 might have higher activity against vancomycin resistant Enterococcus fecium (VRE) strains (compared to vancomycin sensitive strains) was disproved when it was found that VRE strains were also more sensitive to aPDI with Zn-Phe-N5. The minimum inhibitory concentrations of Van-Zn-mPhe-N5 were higher than Van alone, showing antibiotic properties of the Van moiety were lessened in the conjugate. In conclusion Zn-Phe-N5 is a highly active PS against Gram-positive species and deserves further testing. Increasing the number of cationic charges did not have as much effect as expected against Gram-negative species, possibly due to the high molecular weights of the compounds. 